\title{
Dependence of spectral behavior in an InGaN/GaN quantum-well light- emitting diode on the prestrained barrier thickness
}

\author{
Chih-Feng Lu, Chi-Feng Huang, Yung-Sheng Chen, and C. C. Yang ${ }^{\text {a) }}$ \\ Institute of Photonics and Optoelectronics and Department of Electrical Engineering, National Taiwan \\ University, No. 1, Roosevelt Road, Section 4, Taipei 10617, Taiwan
}

(Received 24 February 2008; accepted 23 June 2008; published online 29 August 2008)

\begin{abstract}
The dependencies of output spectral overall redshift and current-density-induced spectral blueshift on the prestrained barrier thickness in the InGaN/GaN quantum-well light-emitting diodes (LEDs) of prestrained growth are demonstrated. Due to the stronger prestrain effect in a sample of a thinner prestrained barrier, the overall spectral redshift range increases and the current-density-induced blueshift range decreases with decreasing prestrained barrier thickness. Also, in terms of device resistance and saturation current, the LED performances of prestrained samples are superior to that of a conventional LED. With a thinner prestrained barrier, the device performance becomes better. The results are attributed to the higher average-indium content and stronger indium-rich clustering behavior in a sample of stronger prestrain. Such attributions are supported by the observations of strain state analysis in transmission electron microscopy measurements. (C) 2008 American Institute of Physics. [DOI: 10.1063/1.2973456]
\end{abstract}

\section{INTRODUCTION}

The quantum-confined Stark effect (QCSE) in an InGaN/ GaN quantum well $(\mathrm{QW})$ plays an important role in the behavior of an InGaN-based light-emitting diode (LED). ${ }^{1,2}$ In this effect, the strong piezoelectric field generated in a well layer results in a potential tilt such that the carrier recombination rate is reduced and the emission spectrum is redshifted. When electric current is injected into the QWs, the carrier screening of the QCSE flattens the potential across a QW leading to a blueshift of LED output spectrum. Recently, we developed a prestrained growth technique for enhancing indium incorporation in an InGaN/GaN QW. ${ }^{3-5}$ In this technique, a low-indium InGaN/GaN QW is grown before the designated light-emitting high-indium InGaN/GaN QWs to create tensile strain in the GaN barrier layer right above the low-indium QW such that the indium incorporation during the growth of the subsequent QWs becomes more effective. Hence, their indium contents can be significantly increased, leading to the efficient emissions of yellow, orange, and red colors. ${ }^{6,7}$ In this situation, the designated light-emitting QWs closer to the low-indium QW receive stronger prestrain effects and have relatively higher indium contents. Because hole migration is much slower than that of electron in nitride compounds, ${ }^{8}$ holes are mainly distributed in the shallower QWs (close to the $p$-type layer) at low injection current levels. As injection current level increases, a higher density of hole can reach the deeper QWs and contribute to longerwavelength emission. This redshift trend in increasing injection current can partly balance the spectral blueshift due to the QCSE screening effect. ${ }^{9}$ It is noted that the aforementioned prestrain effect for enhancing indium incorporation relies on the strength of the tensile strain in the $\mathrm{GaN}$ barrier

\footnotetext{
a) Author to whom correspondence should be addressed. Tel.: 886-2 23657624. FAX: 886-2-23652637. Electronic mail: ccy@cc.ee.ntu.edu.tw.
}

layer right above the low-indium QW. Therefore, it is expected that the prestrain effect depends on the thickness of this barrier layer.

In this paper, we compare three prestrained growth samples of different prestrained $\mathrm{GaN}$ barrier thicknesses with a control sample of no prestrain QW to find that a thinner barrier leads to a more effective prestrain effect of overall spectral redshift and reduced blueshift in increasing injection current. Also, we observe the better device performance, in terms of device resistance and saturation current, in a LED of a thinner prestrained GaN barrier. The observed device behaviors can be well interpreted with the results of transmission electron microscopy (TEM) measurement. In Sec. II of this paper, the epitaxial growth conditions and device fabrication procedures are reported. The device characteristics are discussed in Sec. III. To interpret the device behaviors, the material analysis results are presented in Sec. IV. Finally, conclusions are drawn in Sec. V.

\section{EPITAXIAL GROWTH AND DEVICE FABRICATION}

Both the control and prestrained samples were grown with metalorganic chemical vapor deposition. In the control sample (sample A), first, a $30 \mathrm{~nm}$ GaN nucleation layer was grown on $c$-plane sapphire substrate at $530{ }^{\circ} \mathrm{C}$. Then, after the growth of a $2 \mu \mathrm{m} n$-GaN at $1080{ }^{\circ} \mathrm{C}$, five periods of InGaN/GaN QW with $3 \mathrm{~nm}$ in the well thickness and $15 \mathrm{~nm}$ in the barrier thickness were deposited. In growing the QWs, after the growth of a QW layer at $740{ }^{\circ} \mathrm{C}$, the growth temperature was ramped to $870{ }^{\circ} \mathrm{C}$ within $1 \mathrm{~min}$ for depositing the GaN barrier. After the growth of the QWs, a $30 \mathrm{~nm}$ $p-\mathrm{Al}_{0.2} \mathrm{Ga}_{0.8} \mathrm{~N}$ layer, followed by a $120 \mathrm{~nm} p$-GaN layer (both grown at $945{ }^{\circ} \mathrm{C}$ ), was deposited. All the aforementioned layers were grown at the wafer spin speed of $750 \mathrm{rpm}$. In the prestrained samples, the growth conditions of the LED structure are the same as those of the control sample except that an extra InGaN/GaN QW of lower-indium content 

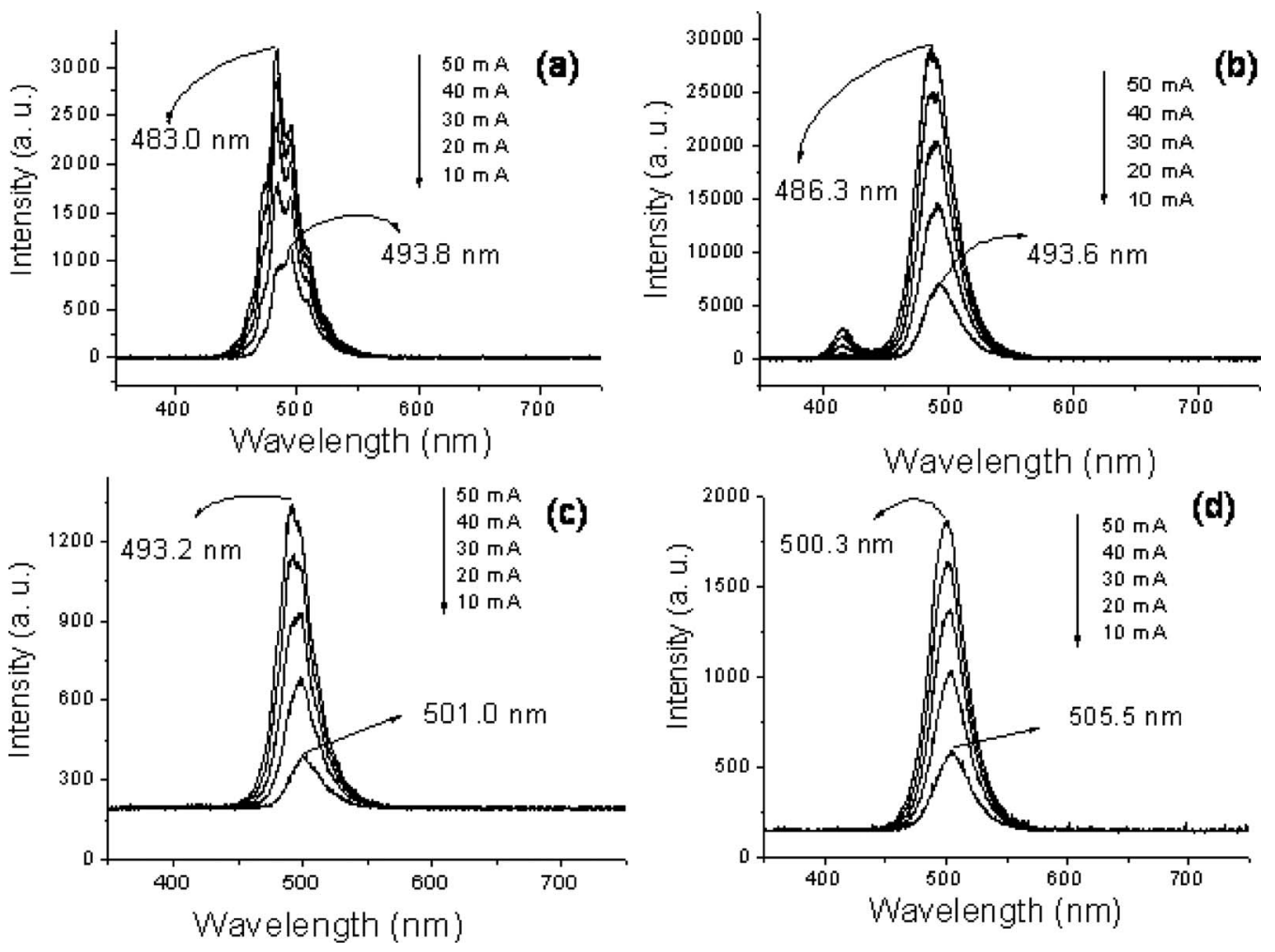

FIG. 1. LED output spectra at various injection current levels of samples A-D in parts (a)-(d), respectively. The spectral peak wavelengths at 10 and $50 \mathrm{~mA}$ in injection current level are shown.

(grown at $750{ }^{\circ} \mathrm{C}$ with the wafer spin speed of $1500 \mathrm{rpm}$ for indium content of about 7\%) was deposited between the $n$-GaN layer and the five higher-indium QWs same as those of the control sample. Three prestrained samples with the thicknesses of the prestrained $\mathrm{GaN}$ barrier at 15,8 , and $5 \mathrm{~nm}$ were prepared and designated as samples $\mathrm{B}, \mathrm{C}$, and D, respectively. LEDs were fabricated based on the four epitaxial samples following the standard process procedures with the mesa size of $300 \times 400 \mu \mathrm{m}^{2}$, which was defined using photolithography and inductively coupled plasma ion etching. Electron-beam evaporation was used to deposit $n$-type Ohmic contact consisting of Ti $(20 \mathrm{~nm}) / \mathrm{Al}(120 \mathrm{~nm}) / \mathrm{Ni}(100$ $\mathrm{nm}) / \mathrm{Au}(150 \mathrm{~nm})$ metal layers. The $p$-type top Ohmic contact consisting of $\mathrm{Ni}(150 \mathrm{~nm}) / \mathrm{Au}(150 \mathrm{~nm})$ metal layers was grown after the deposition of a current spreading layer with $\mathrm{Ni}(5 \mathrm{~nm}) / \mathrm{Au}(5 \mathrm{~nm})$ metal layers. The device was then thermally annealed in nitrogen ambient.

\section{DEVICE CHARACTERISTICS}

Figures 1(a)-1(d) show the LED output spectra at various injection current levels of samples A-D, respectively, indicating variations in spectral feature and intensity. FabryPérot oscillations in the spectra can be seen, particularly in sample A. As marked in the figures, the blueshift ranges of spectral peaks, when the injection current increases from 10 through $50 \mathrm{~mA}$, of samples A through D are 493.8-483.0, 493.6-486.3, 501.0-493.2, and 505.5-500.3 nm, respectively. The blueshifts of $5.2 \mathrm{~nm}$ in sample D, $6.8 \mathrm{~nm}$ in sample C, and $7.3 \mathrm{~nm}$ in sample B are significantly smaller than that of $10.8 \mathrm{~nm}$ in sample A. Because of the FabryPérot oscillations in the spectra, the spectral peak becomes an inappropriate feature for understanding the LED behaviors. In Fig. 2, we show variations in the spectral center of mass in the four samples as functions of injection current. Here, we can first observe that the spectral features of the prestrained samples are generally redshifted when compared with that of the control sample. A thinner prestrained barrier leads to a larger general redshift implying the stronger prestrain effect in such a sample. At the injection current of 50 $\mathrm{mA}$, the spectral center-of-mass redshifts from $2.533 \mathrm{eV}$ of sample A to $2.47 \mathrm{eV}$ of sample $\mathrm{D}$. The second observation in Fig. 2 is that the blueshift range of the control sample is larger than those of the prestrained samples when the injection current increases from 10 through $50 \mathrm{~mA}$. In samples A-D, the blueshifts are $33.8,30.3,30.2$, and $18.9 \mathrm{meV}$, respectively. Generally, a thinner prestrained barrier results in a smaller blueshift range.

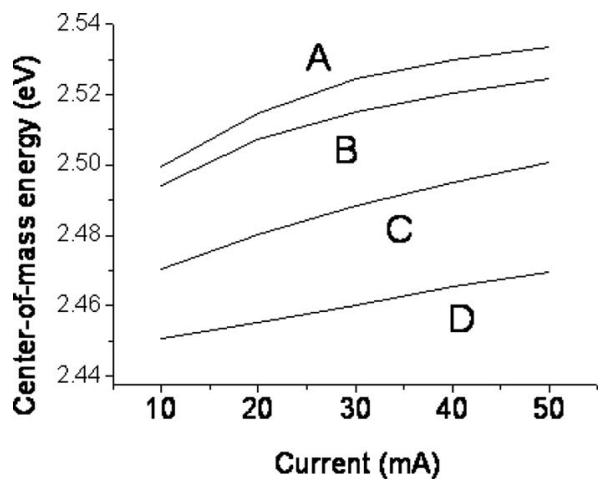

FIG. 2. Variations in the LED spectral center of mass in the four samples when the injection current is increased from 10 through $50 \mathrm{~mA}$. 


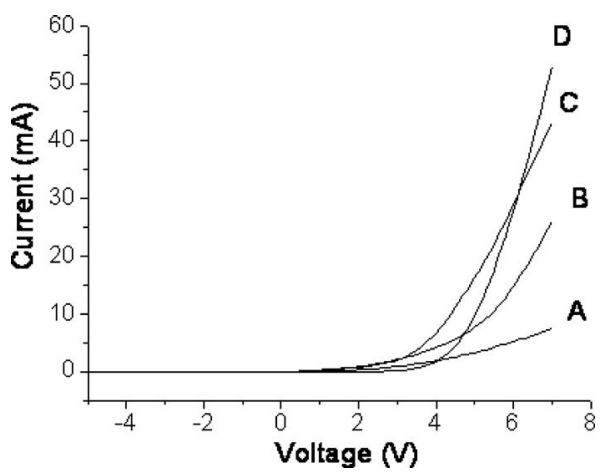

FIG. 3. Current vs voltage curves of the four samples.

Figure 3 shows the injection current variations versus applied voltage of the four samples. Here, one can see that the turn-on voltages of all samples are between 3 and $4 \mathrm{~V}$. However, the device resistance level of the control sample (A) is higher than those of the prestrained samples. The device resistance decreases with decreasing prestrained barrier thickness even though the turn-on voltage of sample $\mathrm{D}$ is slightly higher. The portion of reverse-biased voltage in Fig. 3 indicates that the leakage currents in all the four samples are quite small. Besides the crystal quality issue, the poor electrical property of sample A can be related to the device process quality of this sample. Figure 4 shows the LED output intensities versus injection current in the four samples. Here, it is interesting to see that the output saturation currents of the prestrained samples are higher than that of the control sample. Meanwhile, a thinner prestrained barrier leads to a higher output saturation current level. These trends are generally consistent with those shown in Fig. 3, implying that the prestrain effect may improve the performance of a LED. Also, a thinner prestrained barrier leads to a better performance.

\section{MATERIAL ANALYSIS}

To understand the indium content variations among different QWs in those samples, we performed high-resolution TEM experiment on the four samples. Based on the atomiclevel images, we used the algorithm of the strain state analysis (SSA) (Ref. 4, 10, and 11) to obtain the strain mapping and hence indium atom distribution around a $\mathrm{QW}$ layer for calibrating the average-indium content of the QW. Although

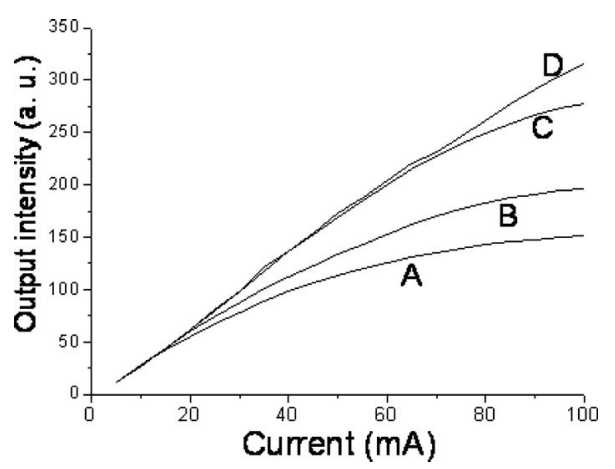

FIG. 4. Variations in the LED output intensity vs injection current of the four samples.
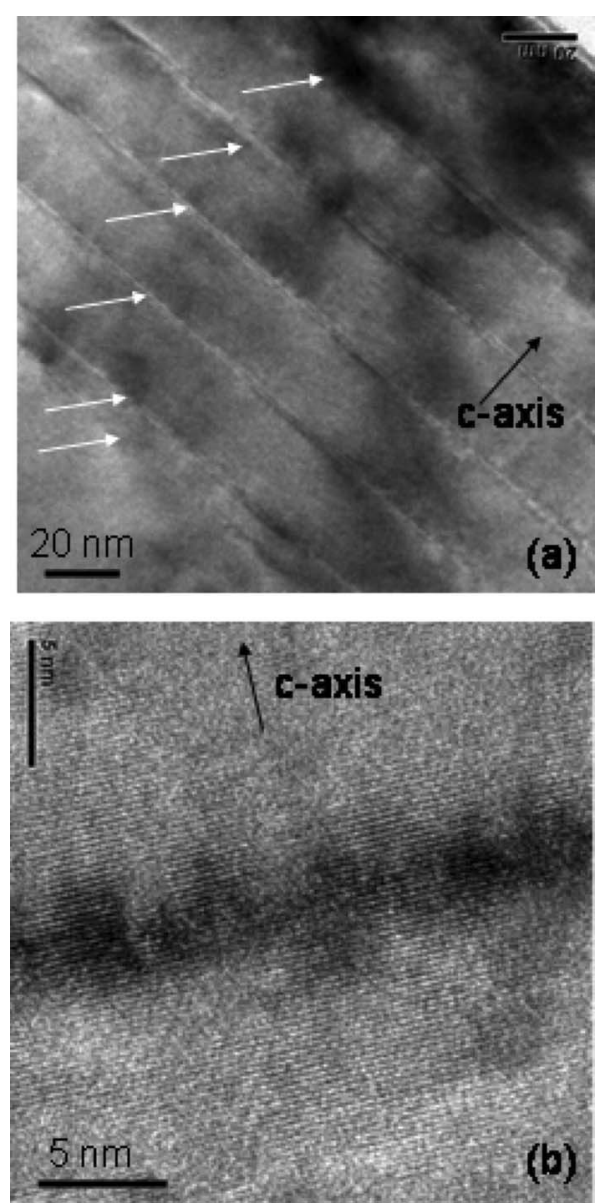

FIG. 5. Cross-section TEM images of sample D. (a) An image showing the six QWs (indicated by arrows) with the low-indium one at the lower-left corner and (b) a two-electron-beam image of the third QW from the top.

such results cannot be very accurate, they can provide us with a general trend of indium content variation. ${ }^{4}$ Figure 5 shows two cross-section TEM images of sample D, including part (a) for illustrating the six QWs (indicated by arrows) with the low-indium one at the lower-left corner and (b) for a two-electron-beam image of the third QW from the top. Although the locations and shapes of the six QWs are well defined in the low-resolution image of Fig. 5(a), nonuniform indium distribution around the designed QW layer can be seen in the higher-resolution image of Fig. 5(b). ${ }^{12}$ The twoelectron-beam images such as Fig. 5(b) for all the QWs of the four samples are used for SSA. Figures 6(a) and 6(b) show the SSA images of the sixth and third QWs, respectively, from the top of sample D. Here, different colors represent different indium compositions, as shown in the legend. The sixth QW corresponds to the low-indium one and hence results in a quite uniform and low-indium distribution. The third QW shows quite strong indium composition fluctuation, as featured by the red spot, which corresponds to an indiumrich cluster. Based on the indium composition distributions such as those in Figs. 6(a) and 6(b), the average-indium content of each QW can be evaluated. ${ }^{4}$ The average-indium contents of the sixth and third QWs in Figs. 6(a) and 6(b) are $7.22 \%$ and $11.28 \%$, respectively. Figure 7 shows variations in average-indium content among various QWs in the four samples. The sequence of $\mathrm{QW}$ is counted from the top 


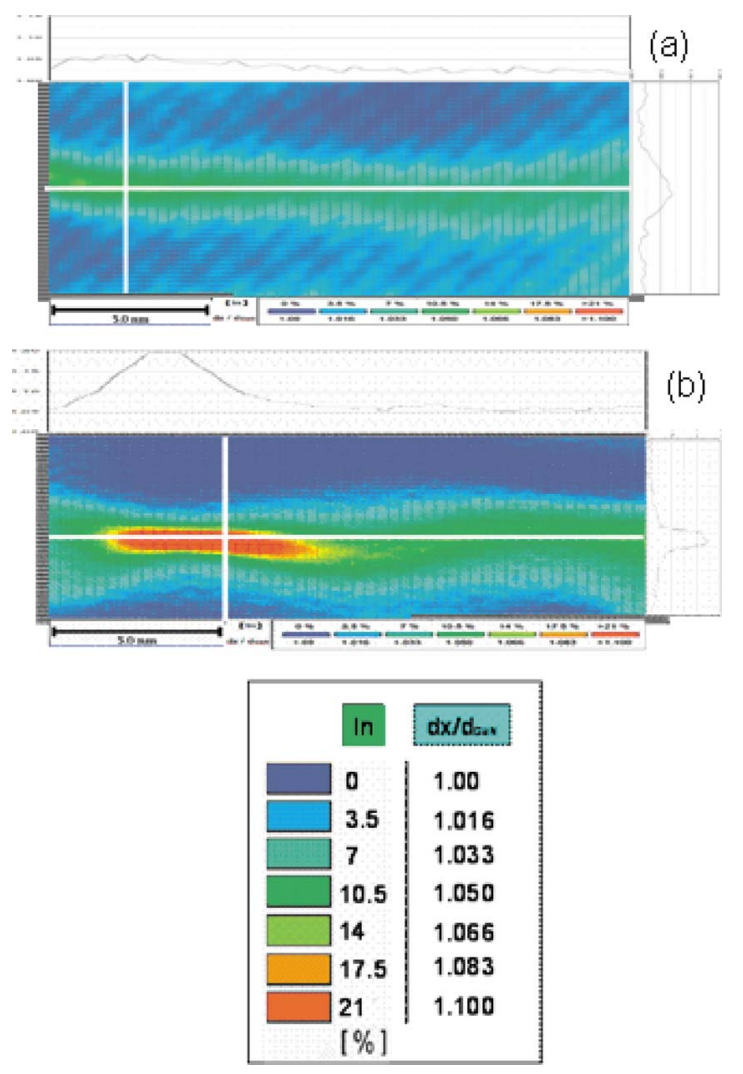

FIG. 6. (Color online) (a) SSA images of the low-indium QW and (b) the third QW from the top of sample D. The legend at the figure bottom shows the color coding for various indium (In) compositions and the corresponding strain levels $d x / d_{\mathrm{GaN}}$. Here, $d_{\mathrm{GaN}}$ is the lattice constant of $\mathrm{GaN}$ and $d x$ is the deviation of lattice constant in InGaN from $d_{\mathrm{GaN}}$.

(p-type side), and hence QW 6 corresponds to the lowindium QW for generating the prestrain effect. Here, one can see the general increase in indium content in the prestrained samples (except sample B) that is consistent with the variation trend of LED output spectrum shown in Fig. 2. Again, a thinner prestrained barrier results in generally higher indium content. The decreasing trend of indium content in growing more QWs can also be seen, which is consistent with the previously reported results. ${ }^{3-5}$

With a thinner prestrained barrier, the tensile strain applied on the barrier layer by the low-indium QW is expected to be stronger and hence the indium contents of the subse-

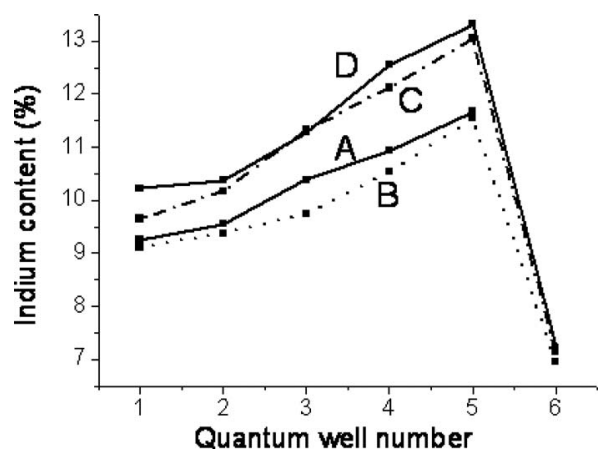

FIG. 7. Calibrated average-indium contents of various QWs in the four samples. The QW sequence is counted from the top surface (the p-type side). quently grown QWs are higher, leading to the larger general redshift of output spectrum, as shown in Fig. 2. With the more significant increase in indium incorporation, the contrast of indium content between QWs 1 and 5 is expected to be generally larger. Hence, as injection current increases and more holes are supplied to the deeper QWs, the spectral redshift trend is more significant for balancing the blueshift trend due to QCSE screening. Although the QCSE screening effect can be stronger in a sample of thinner prestrained barrier, the more significant redshift trend can effectively reduce the overall blueshift range. It is noted that the LED output spectral width does not significantly increase even though the indium contents among different QWs are different. The spectral full widths at half maximum of samples A-D are $30.4,31.2,26.4$, and $31.6 \mathrm{~nm}$ at $10 \mathrm{~mA}$ and $31.5,34.8,31.6$, and $34.4 \mathrm{~nm}$ at $50 \mathrm{~mA}$, respectively. The increases in spectral widths in increasing injection current are quite small. It is speculated that at a fixed injection current level, the LED output is mainly contributed from part of the QWs. The better performances of the prestrained samples, when compared with the control sample, can be partly attributed to the higher-indium-rich cluster densities and composition contrasts under the condition of prestrained growth. It has been shown with the aforementioned SSA that the indium-rich clustering behavior in a stronger prestrained sample is more significant. From the SSA images, we can estimate the indium composition fluctuation ranges (from crests to valleys) along the QW layers. The estimated fluctuation ranges of samples A-D are $14 \%-18 \%, 15 \%-25 \%, 17 \%-37 \%$, and $18 \%-49 \%$, respectively, indicating that a stronger prestrained sample indeed has a stronger clustering behavior. Such a cluster structure can lead to better carrier confinement for recombination and hence better device performance, including higher saturation current. ${ }^{4,13}$ Other possible mechanisms for the better performances of the prestrained samples deserve more studies.

Compared with the previously reported results of prestrained growth, the induced redshift via the increased indium incorporation of this work is smaller. In this work, in the injection current at $10 \mathrm{~mA}$, the electroluminescence (EL) spectral peak redshifts from $2.511 \mathrm{eV}$ or $493.8 \mathrm{~nm}$ of sample A (unprestrained) to $2.453 \mathrm{eV}$ or $505.5 \mathrm{~nm}$ of sample D that shows a redshift of $58 \mathrm{meV}$ or $11.7 \mathrm{~nm}$. However, in Ref. 3, also at $10 \mathrm{~mA}$, the EL spectral peak redshifts from $2.292 \mathrm{eV}$ or $541 \mathrm{~nm}$ in the control sample to $2.084 \mathrm{eV}$ or $595 \mathrm{~nm}$ in the prestrained sample that means a redshift of $208 \mathrm{meV}$ or 54 $\mathrm{nm}$. The comparison shows that the prestrained growth leads to a larger spectral redshift when the indium content of the unprestrained QW is higher. Without the prestrain effect, the QW indium incorporation can be increased by lowering the growth temperature. Based on this and the previously reported prestrained growth results, ${ }^{3,6,9}$ we can conclude that the prestrain-induced indium-incorporation enhancement is more significant at a lower growth temperature. The prestrained growth produces tensile strains in the GaN barriers to make indium incorporation more effective and to partially relax the strain in a subsequently grown $\mathrm{InGaN} / \mathrm{GaN}$ QW. At a lower growth temperature, the higher-indium content can lead to more significant strain relaxation through the 
spinodal decomposition process for forming strong composition fluctuations and indium-rich clusters. ${ }^{12,14}$ In this situation, even higher-indium incorporation can be achieved under the prestrain condition. Therefore, originally higherindium content can lead to a more significant prestrain effect or a larger spectral redshift. In the visible range, the trend of increasing prestrain effect through lowering the growth temperature can be correct. However, up to a certain point, at which the indium-rich cluster density reaches a critical level, this trend is expected to stop. Nevertheless, such a speculation requires confirmation.

\section{CONCLUSIONS}

In summary, we have demonstrated the dependencies of output spectral overall redshift and spectral blueshift in increasing injection current on the prestrained barrier thickness in an InGaN/GaN QW LED of prestrained growth. It was found that a thinner prestrained barrier led to a larger general spectral redshift and a smaller blueshift in increasing injection current because of the stronger prestrain effect. Also, it was found that in terms of device resistance and saturation current, the LED performances of prestrained samples were better than that of a conventional LED. A LED of a thinner prestrained barrier had a better performance. These observations were attributed to the higher average-indium content and stronger indium-rich clustering behavior in a sample of stronger prestrain. These attributions were supported by the SSA results in the TEM measurements.

\section{ACKNOWLEDGMENTS}

This research was supported by the National Science Council, Republic of China, under Grant Nos. NSC 96-2120M-002-008 and NSC 96-2628-E-002-044-MY3, and by the U.S. Air Force Scientific Research Office under Contract No. AOARD-07-4010.

${ }^{1}$ S. F. Chichibu, A. C. Abare, M. S. Minsky, S. Keller, S. B. Fleischer, J. E. Bowers, E. Hu, U. K. Mishra, L. A. Coldren, S. P. DenBaars, and T. Sota, Appl. Phys. Lett. 73, 2006 (1998).

${ }^{2}$ P. Riblet, H. Hirayama, A. Kinoshita, A. Hirata, T. Sugano, and Y. Aoyagi, Appl. Phys. Lett. 75, 2241 (1999).

${ }^{3}$ C. F. Huang, T. Y. Tang, J. J. Huang, W. Y. Shiao, C. C. Yang, C. W. Hsu, and L. C. Chen, Appl. Phys. Lett. 89, 051913 (2006).

${ }^{4}$ Y. S. Chen, L. J. Yao, Y. L. Lin, L. Hung, C. F. Huang, T. Y. Tang, J. J. Huang, W. Y. Shiao, and C. C. Yang, J. Cryst. Growth 297, 66 (2006).

${ }^{5}$ W. Y. Shiao, C. F. Huang, T. Y. Tang, J. J. Huang, and C. C. Yang, J. Appl. Phys. 101, 113503 (2007).

${ }^{6}$ C. F. Huang, T. Y. Tang, J. J. Huang, and C. C. Yang, Appl. Phys. Lett. 90, 151122 (2007).

${ }^{7}$ H. S. Chen, C. F. Lu, D. M. Yeh, C. F. Huang, J. J. Huang, and C. C. Yang, IEEE Photonics Technol. Lett. 18, 2269 (2006).

${ }^{8}$ S. Fujita, M. Funato, D. C. Park, Y. Ikenaga, and S. Fujita, MRS Internet J. Nitride Semicond. Res. 4S1, G6.31 (1999).

${ }^{9}$ C. F. Huang, C. Y. Chen, C. F. Lu, and C. C. Yang, Appl. Phys. Lett. 91, 051121 (2007).

${ }^{10}$ D. Gerthsen, B. Neubauer, A. Rosenauer, T. Stephan, H. Kalt, O. Schon, and M. Heuken, Appl. Phys. Lett. 79, 2552 (2001).

${ }^{11}$ A. Rosenauer, U. Fischer, D. Gerthsen, and A. Forster, Appl. Phys. Lett. 71, 3868 (1997).

${ }^{12}$ Y. S. Lin, K. J. Ma, C. Hsu, Y. Y. Chung, C. W. Liu, S. W. Feng, Y. C. Cheng, M. H. Mao, C. C. Yang, H. W. Chuang, C. T. Kuo, J. S. Tsang, and T. E. Weirich, Appl. Phys. Lett. 80, 2571 (2002).

${ }^{13}$ Y. C. Cheng, E. C. Lin, C. M. Wu, C. C. Yang, J. R. Yang, A. Rosenauer, K. J. Ma, S. C. Shi, L. C. Chen, C. C. Pan, and J. I. Chyi, Appl. Phys. Lett. 84, 2506 (2004).

${ }^{14}$ I.-h. Ho and G. B. Stringfellow, Appl. Phys. Lett. 69, 2701 (1996). 